\title{
PRINTED-CIRCUIT MAGNETS FOR THE UNIVERSITY OF MARYLAND ELECTRON RING (UMER) — NEW DEVELOPMENTS*
}

\author{
H. Li ${ }^{\dagger}$, S. Bernal, R.A. Kishek, T. Godlove, P.G. O'Shea, M. Reiser \\ Institute for Research in Electronics and Applied Physics, University of Maryland, \\ College Park, MD 20742
}

\begin{abstract}
The short printed-circuit quadrupoles for UMER [see invited paper by P.G. O'Shea] produce fields with small gradients that meet our needs for the focusing and bending of a $10 \mathrm{keV}, 100 \mathrm{~mA}$ electron beam over a circumference of $11.5 \mathrm{~m}$. The field quality of the magnets has been established [see W.W. Zhang et al., Phys. Rev. ST Accel. Beams 3, 122401 (2000)], but correcting elements are needed to compensate for quadrupole rotation errors that lead to undesirable beam rotations in the injector line as well as in the main lattice. The correcting elements consist of printed-circuit quadrupoles that are mounted over the main quadrupoles, with a relative angle of 45 degrees. In this way, a skew quadrupole component can be easily, independently and electronically adjusted to rotate the quadrupole field. Results are presented of field measurements of the modified quadrupoles, as well as preliminary observations in the UMER injector, and corresponding computer simulations.
\end{abstract}

\section{INTRODUCTION}

Air-core printed-circuit (PC) quadrupoles have been developed for the University of Maryland Electron Ring $[1,2]$, to focus a $10 \mathrm{keV}, 100 \mathrm{~mA}$ electron beam over a circumference of $11.5 \mathrm{~m}$. The printed-circuit currents generate a quadrupole field with small gradient and high quality [3]. However, rotation errors about the magnet axis, resulting from assembly and mounting, can be detrimental to the beam quality. The beam can be rotated, leading to beam distortions and mismatch, with a net increase in beam emittance [4,5]. Thus, a new type of magnet, called PC skew quadrupole, has been developed to compensate for the errors. We describe here the design and preliminary tests of the new magnets.

\section{PC SKEW QUADRUPOLE DESIGN}

As it is well known, the cylindrical components of the magnetic field of a quadrupole are given by

$$
B_{r}=g_{0} r \sin 2 \theta, \quad B_{\theta}=g_{0} r \cos 2 \theta,
$$

where $g_{0}$ is the field gradient.
$B_{r}$ and $B_{\theta}$, are the so-called normal components. The corresponding skew components are introduced by a quadrupole rotation in the $\theta$ direction. Bellow, we concentrate on $B_{\theta}$, but similar expressions apply to $B_{r}$. If $\alpha$ is the quadrupole rotation angle, the rotated field $B_{\theta}$ is given by

$$
\begin{aligned}
& B_{\theta}=g_{0} r \cos 2(\theta-\alpha)=B_{n}+B_{s}, \\
& B_{n}=\cos (2 \alpha) g_{0} r \cos (2 \theta), \\
& B_{s}=\sin (2 \alpha) g_{0} r \sin (2 \theta),
\end{aligned}
$$

where $B_{n}$ and $B_{s}$ are the normal and skew quadrupole components, respectively. Usually, the rotation $\alpha$ is small so that $B_{s}$ is very small compared with $B_{n}$, which further simplify the formulas as

$$
B_{n}=g_{0} r \cos (2 \theta), B_{s}=2 \alpha g_{0} r \sin (2 \theta) .
$$

In designing a PC skew quadrupole, we use two pairs of printed circuits, one of which is the normal PC, and the other one is the skew PC. The latter is mounted over the main PC with a $45^{\circ}$ rotation. Figure 1 illustrates the relative placement of the skew and normal quadrupoles, while Figure 2 shows a photo of a disassembled main PC quadrupole together with the added skew quadrupole. The two PCs are attached closely together and to an aluminum mandrel.

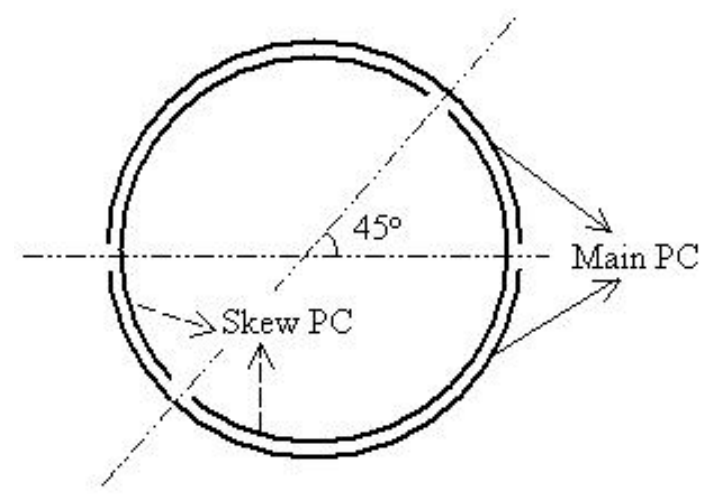

Figure 1: Layout of PC Skew quadrupole and Normal (Main) PC quadrupole.

\footnotetext{
* Research supported by the U.S. Department of Energy.

$\dagger$ lihui@glue.umd.edu
} 


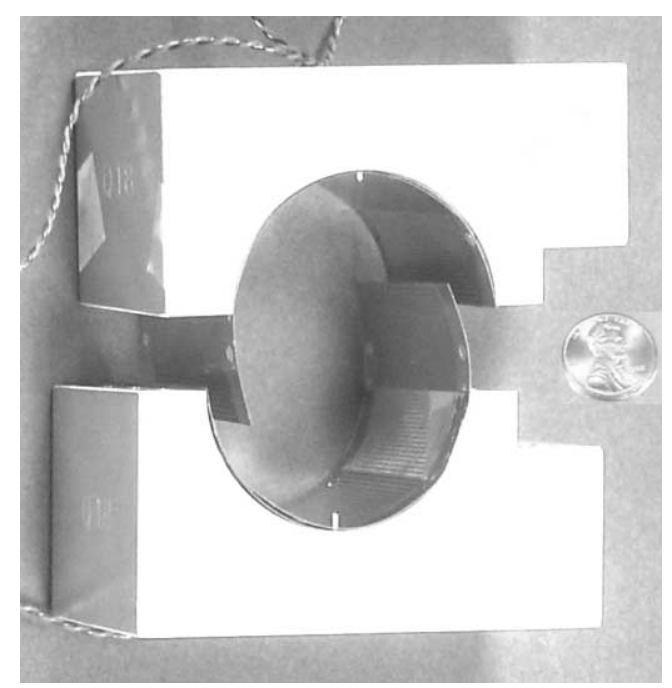

Figure 2: Main and skew PC quadrupole assembly.

The main and skew quadrupoles are powered by different current supplies, so that the normal and skew components can be independently adjusted. It is a very convenient design, since we only need to set up a small current to the skew PC in order to compensate for a possible mechanical rotation error of the main quadrupole. This avoids the need for direct mechanical corrections, which would be very difficult in our case.

\section{FIELD MEASUREMENTS}

We have accurately measured the field quality of the main PC quadrupoles with a rotating coil magnetometer [3]. Using the same rotating coil apparatus and similar techniques, we have characterized the fields of the new main + skew quadrupole assembly. We usually supply a large current to the main PC, and very small currents to the skew PCs in the experiment. The skew quadrupole introduces a phase shift in the observed quasi-sinusoidal induced voltage, which can be measured with high accuracy. Table 1 shows the rotation angle introduced by the skew PCs with currents ranging from $20 \mathrm{~mA}$ to 100 $\mathrm{mA}$, corresponding to $1 \%-5 \%$ of the normal current (2A). The calculated values for the phase angle (see Eq. 3) are in good agreement with measurements.

Table 1: Calculated vs. measured rotation angle

\begin{tabular}{|c|c|c|c|c|c|}
\hline $\begin{array}{c}\text { Skew current } \\
\text { mA }\end{array}$ & 20 & 40 & 60 & 80 & 100 \\
\hline $\begin{array}{c}\text { Rotation angle } \\
\text { (theory) deg }\end{array}$ & 0.29 & 0.57 & 0.86 & 1.15 & 1.43 \\
\hline $\begin{array}{c}\text { Rotation angle } \\
\text { (measure) deg }\end{array}$ & 0.29 & 0.65 & 0.93 & 1.24 & 1.52 \\
\hline
\end{tabular}

Measurements of multipole content of the modified quadrupole with the same skew currents as in Table 1 show that the amplitude of all higher order multipoles (sextupole, octupole, etc.) are smaller than one percent of the quadrupole component. This is similar to our previous results reported in Ref. [3] for the standard PC quadrupoles.

\section{EXPERIMENT AND SIMULATION}

We performed preliminary tests of the skew quadrupole in the UMER injector line [6]. The straight part of the injector line has four quadrupoles that we label as "Q1" through "Q4". The quadrupole halves are clamped to a common plate (flat to within $0.01 \mathrm{~mm}$ ), so the rotation errors from mounting are minimized. In fact, the beam rotations that are observed in the matching experiments are small, and may be the result of residual errors from assembly of the printed circuits on the aluminum mounts. In UMER, however, the relative rotations of groups of quadrupoles, each group (2 FODO periods each) sharing a support plate, may be more problematic.

Therefore, in order to gain a better understanding of the effects of rotated quadrupoles in UMER, and to explore general issues of beam transport dynamics with rotation errors, we have designed a controlled experiment. The first quadrupole, Q1, is fitted with a skew quadrupole as in Fig. 2. The normal current of the main quadrupole is $1.545 \mathrm{~A}$. The skew quadrupole current is varied from $0.1 \mathrm{~A}$ to $0.5 \mathrm{~A}$, corresponding to rotation errors from $1.86^{\circ}$ to $9.44^{\circ}$. Simultaneously, the main currents are also reduced from $1.545 \mathrm{~A}$ to $1.462 \mathrm{~A}$ to keep the amplitude of the net quadrupole field constant. Figure 3(a) shows fluorescent screen pictures taken along the injector line when the skew and main currents are $0.2 \mathrm{~A}$ and $1.532 \mathrm{~A}$, respectively. This current setup yields a $3.72^{\circ}$ rotation angle of the quadrupole field in Q1. In Fig. 3 some of the beams appear truncated because of a limited phosphor screen size.

For comparison, simulated beam pictures, based on the particle-in-cell code WARP, are shown in Fig. 3(b). As seen in the figure, the pictures show good agreement regarding beam rotations and shapes, despite some differences between the particle distributions.

The rotation angles of the beam cross sections along the injector line can be expressed in terms of second order moments of the electron distributions. For the simulation and experimental photos, we use the following formula, derived by Barnard [4]:

$$
\tan 2 \alpha=\frac{2 \Delta x y}{\Delta x^{2}-\Delta y^{2}}
$$

where $\Delta x y=\langle x y\rangle-\langle x\rangle\langle y\rangle$ and \langle\rangle indicates average over particles [4].

Figures 4 and 5 show results of experiments vs. simulations for the same locations as Fig 3. At Q2, the beam has rotated by $3.8^{0}$ (Fig. 3, and Fig.5, solid curve), almost the same as the rotation error in Q1. At Q3, the beam rotation angle is about $9.7^{0}$, relative to the horizontal (the un-rotated orientation), while at Q4 almost the same angle is measured relative to the vertical. Similar considerations apply for the other skew currents. 


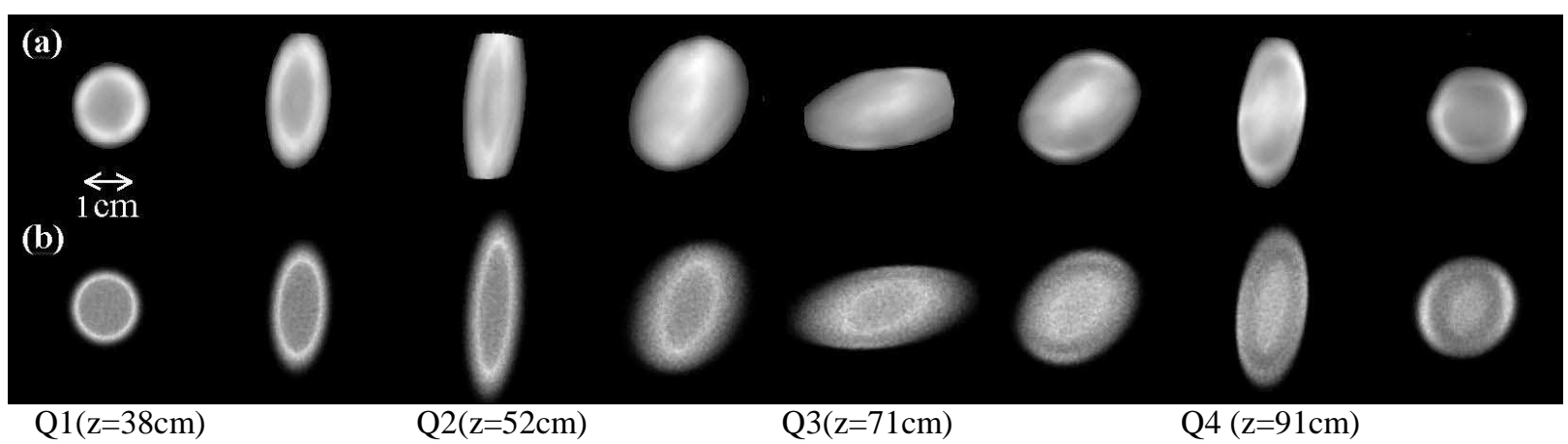

Figure 3: Effects of rotated first quadrupole Q1 in UMER injector: (a) Phosphor screen pictures, and (b) WARP simulations. The quadrupole field of Q1 is rotated $3.72^{\circ}$ by using a skew current of $0.2 \mathrm{~A}$.

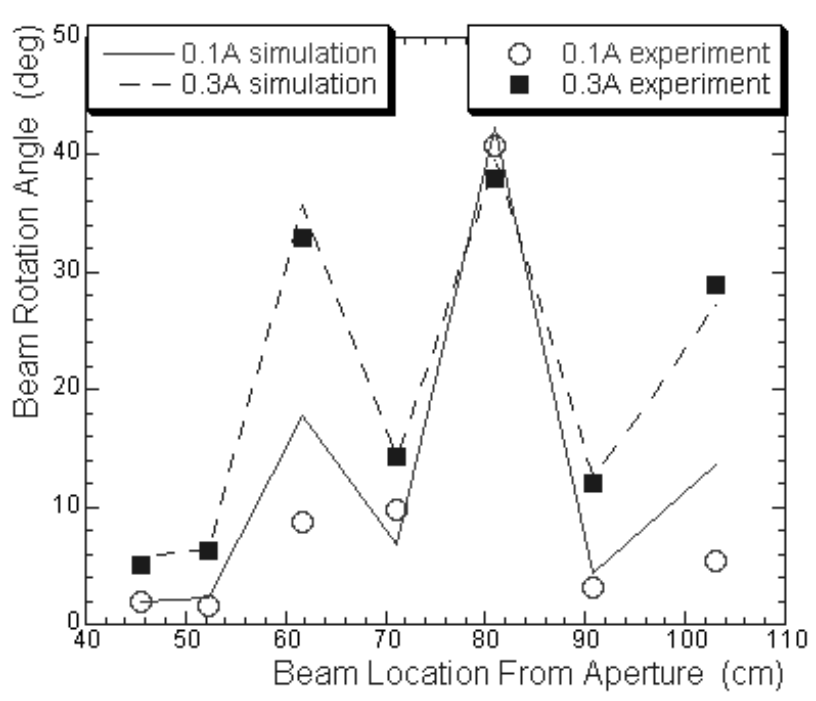

Figure 4: Beam rotation angle along injector: experiment vs. simulation. Skew Currents $=0.1$ and $0.3 \mathrm{~A}$

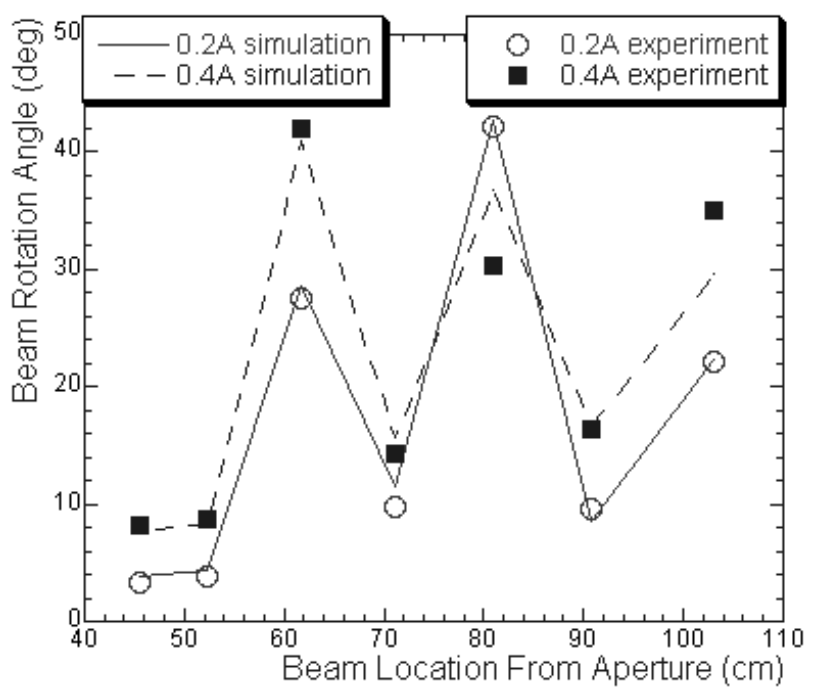

Figure 5: Beam rotation angle along injector:

experiment vs. simulation. Skew Currents $=0.2$ and $0.4 \mathrm{~A}$
Good agreement between simulations and experiment is seen. Errors can be traced to image calibration ( $\mathrm{mm} / \mathrm{pixel}$ in the digitized pictures) and possible small effects from image forces not included in Eq. (4).

\section{CONCLUSIONS}

The Skew PC quadrupoles have been successfully designed and implemented in the UMER injector. The good agreement between preliminary experiments and simulations not only proves the use of skew quadrupoles for correcting quadrupole rotation errors, but also provides us with a convenient and easy tool to explore beam rotation dynamics. Future experiments will explore the effects of random rotation errors for a closer comparison with existing simulations.

\section{REFERENCES}

[1]http://www.ireap.umd.edu/umer

[2]P.G. O'Shea et al, The University of Maryland Electron Ring (UMER) (Invited), TOAA008, these proceedings.

[3] W.W. Zhang et al., Design and field measurement of printed-circuit quadrupoles and dipoles, Phys. Rev. ST Accel. Beams 3, 122401 (2000).

[4]John J. Barnard, Emittance Growth from Rotated Quadrupoles in Heavy Ion Accelerators, Proc. 1995 Particle Accel. Conf. 3241, (1996).

[5]R.A. Kishek et al, Effects of Quadrupole Rotations on the Transport of Space-Charge-Dominated Beams: Theory and Simulations Comparing Linacs with Circular Machines, Proc. 1999 Particle Accel. Conf. , 1761 (1999). [6]S. Bernal et al, Beam Tests of the $10 \mathrm{keV}$ Injector for the UMER, WPAH023, these proceedings. 